\title{
Relationship between dietary patterns and lung function in young adults: the Young Hearts Project, Northern Ireland
}

\author{
C. R. Whittle ${ }^{1}$, H. J. McCourt ${ }^{1}$, I. S. Young ${ }^{1}$, L. J. Murray ${ }^{2}$, C. A. Boreham ${ }^{3}$, C. E. Neville ${ }^{1}$, \\ A. M. Gallagher ${ }^{4}$, C. R. Cardwell ${ }^{2}$, J. V. Woodside ${ }^{1}$ and M. C. McKinley ${ }^{1}$ \\ ${ }^{1}$ Nutrition and Metabolism Group, Centre for Public Health, Queen's University Belfast, Belfast, BT12 6BJ, \\ ${ }^{2}$ Cancer Epidemiology and Health Services Group, Centre for Public Health, Queen's University Belfast, BT12 6BJ, \\ ${ }^{3}$ Institute for Sport and Health, University College Dublin, Dublin, Ireland and ${ }^{4}$ Northern Ireland Centre for Food \\ and Health (NICHE), University of Ulster, Coleraine, BT52 ISA, UK
}

Studies of individual nutrients and foods have revealed much about dietary influences on respiratory health. Prospective studies have shown that diets rich in fruit, vegetables, antioxidants, wholegrains and $n-3$ PUFA in oily fish are associated with a higher level of lung function $^{(1,2)}$. Given these findings and the complexity of factors reported to affect lung function, it is likely to be influenced by a wide range of nutrients. Multiple food or nutrient approaches, such as the application of predefined a priori dietary quality scores, are now widely used in nutritional epidemiology. Such approaches are said to better reflect the complex interactions between nutrients or foods that are not adequately accounted for when examining isolated nutrients ${ }^{(3)}$. The aim of the present study was to examine the relationship between lung function and dietary patterns in young adults who participated in the Young Hearts Project, Northern Ireland.

The Young Hearts Project $(\mathrm{YH})$ is a longitudinal observational study initially designed to investigate coronary risk factors in $12-15$ year olds $^{(4)}$. The current findings are based on Young Hearts 3 (YH3) ${ }^{(5)}$, a follow-up study carried out between 1997-1999 (48.2\% response rate; $n=489,51 \%$ men) when the participants were aged 20-25 years old. Ethical approval was obtained from the Research Ethics Committee, Queen's University Belfast, and written informed consent was obtained from all participants. Spirometry testing measured forced vital capacity $(\mathrm{FVC})$ and forced expiratory volume in one second $\left(\mathrm{FEV}_{1}\right)$ in a standardised manner. Diet was assessed using a 7-day diet history and analysed using a computerised dietary analysis programme. Three validated $a$ priori dietary quality scores were applied to the dietary data: dietary diversity score (DDS), a simple food group based score with a potential range from 0-5; nutritional risk score (NRS), a nutrient based ranking index corresponding to the intake of 19 individual nutrients with potential range from 1-489 and Mediterranean Diet Score (MDS), which is based on a whole dietary pattern that is known to be associated with reduced concentrations of CVD risk biomarkers, with a potential range from 0-9. In each case, higher scores represent a more optimal diet. Associations between the dietary quality scores (categorised into tertiles) and lung function were assessed using multivariate linear regression, adjusting for the confounding factors age, BMI, smoking status, physical activity, socioeconomic status and energy intake. The mean (SD, range) for each of the three dietary scores is as follows: Men DDS 2.1 (0.4, 1.0-3.0), NRS 126.0 (19.5, 43.4-169.7), MDS 3.7 (0.9, 0.8-6.0); and women - DDS 2.0 (0.4, 1.0-2.9), NRS 119.5 (19.5, 48.7-163.7), MDS 3.7 (0.9, 1.1-6.1).

Men had significantly greater $\mathrm{FEV}_{1}$ with increasing MDS $(P=0.040)$. Similarly, a trend towards greater FVC in men and FEV $\mathrm{F}_{1}$ in women was observed with increasing $\operatorname{MDS}(P=0.055$ and $P=0.069$ respectively). In contrast men with a greater DDS had a trend towards a lower $\mathrm{FEV}_{1} / \mathrm{FVC}$ ratio $(P=0.052)$. No relationships were identified between DDS in women and NRS in men or women and lung function.

In conclusion adherence to a Mediterranean diet may be beneficial for lung function in young adults.

The Young Hearts Project, Northern Ireland was supported by the Wellcome Trust and the British Heart Foundation.

1. Varraso R, Fung TT, Hu FB et al. (2007) Am J Clin Nutr 86, 488-495.

2. Varraso R, Fung TT, Hu FB et al. (2007) Thorax 62, 786-791.

3. Hu (2002) Curr Opin Lipidol 13, 3-9.

4. Boreham C, Savage JM, Primrose D et al. (1993) Archives of Disease in Childhood; 68, 182-186.

5. Gallagher AM, Savage JM, Murray LJ et al. (2002) Public Health 116, 332-340. 